\title{
Correction to: A continuation method for spatially discretized models with nonlocal interactions conserving size and shape of cells and lattices
}

\author{
Shin-Ichiro $\mathrm{Ei}^{1} \cdot$ Hiroshi Ishii ${ }^{1} \cdot$ Makoto Sato $^{2} \cdot$ Yoshitaro Tanaka $^{3}$ (D) \\ Miaoxing Wang ${ }^{2} \cdot$ Tetsuo Yasugi $^{2}$
}

Published online: 8 May 2021

๑) Springer-Verlag GmbH Germany, part of Springer Nature 2021

\section{Correction to: Journal of Mathematical Biology (2020) 81:981-1028 https://doi.org/10.1007/s00285-020-01534-6}

The original version of this article unfortunately contained a mistake in Fig. 1b. The corrected Fig. $1 \mathrm{~b}$ is given below.

The original article can be found online at https://doi.org/10.1007/s00285-020-01534-6.

\footnotetext{
$凶$ Yoshitaro Tanaka

yoshitaro.tanaka@gmail.com

$\bowtie \quad$ Tetsuo Yasugi

tetsuo.yasugi@staff.kanazawa-u.ac.jp

Shin-Ichiro Ei

Eichiro@math.sci.hokudai.ac.jp

Makoto Sato

makotos@staff.kanazawa-u.ac.jp

1 Department of Mathematics, Faculty of Science, Hokkaido University, Sapporo 060-0810, Japan

2 Mathematical Neuroscience Unit, Institute for Frontier Science Initiative, Kanazawa University, 13-1 Takaramachi, Kanazawa-shi, Ishikawa 920-8640, Japan

3 Department of Complex and Intelligent Systems, Future University Hakodate, 116-2 Kamedanakano-cho, Hakodate, Hokkaido 041-8655, Japan
} 


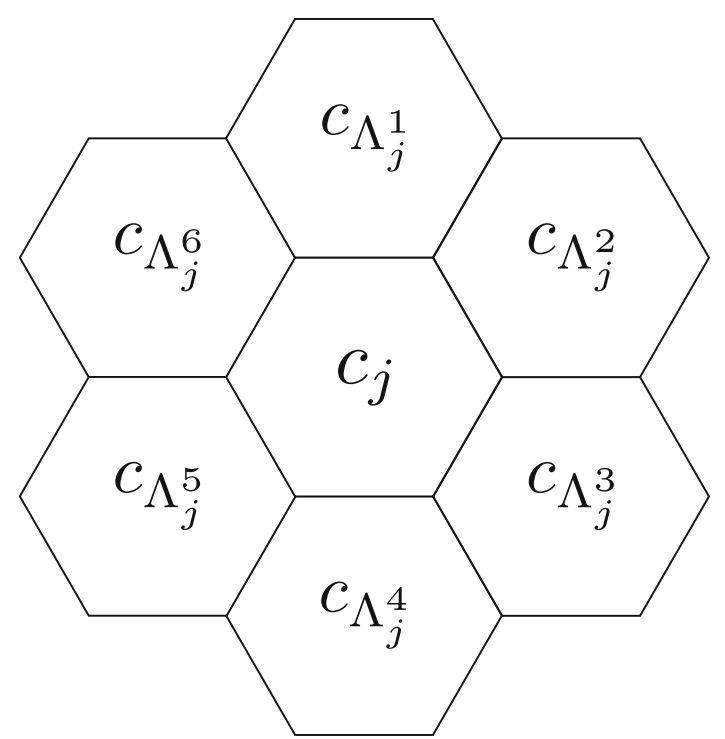

Publisher's Note Springer Nature remains neutral with regard to jurisdictional claims in published maps and institutional affiliations. 